\title{
DESIGN, DEVELOPMENT AND CHARACTERIZATION OF PACLITAXEL LOADED SOLID LIPID NANOPARTICLES AS A COLLOIDAL DRUG CARRIER
}

\author{
INDRAYANI D. RAUT ${ }^{1 *}$, AREHALLI S. MANJAPPA ${ }^{2}$, SHRINIVAS K. MOHITE ${ }^{1}$, RAJENDRA C. DOIJAD ${ }^{3}$
}

${ }^{1}$ Rajarambapu College of Pharmacy, Kasegaon (India), ${ }^{2}$ Tatyasaheb Kore College of Pharmacy, Warananagar (India), ${ }^{3}$ KIMSDU's Krishna Institute of Pharmacy, Karad (India)

Email: idraut7363@gmail.com

Received: 04 Dec 2018, Revised and Accepted: 24 May 2019

\section{ABSTRACT}

Objective: This study was aimed to design and characterize Paclitaxel-loaded Solid Lipid Nanoparticles (SLNs) to achieve site specificity,reduce toxicity and sustained release pattern.

Methods: Paclitaxel-loaded solid lipid nanoparticles were fabricated by microemulsion followed by probe sonication technique using stearic acid as lipid and stabilized of the mixture of surfactants. In this study, $3^{2}$ full factorial design was employed for optimizing the concentration of lipid as stearic acid and surfactant (soya lecithin) for the nanoparticles. The optimization was done by studying the dependent variable of particle size and $\%$ entrapment efficiency.

Results: The results showed that the paclitaxel-loaded solid lipid nanoparticles prepared with the concentration of $33.31 \%$ stearic acid and $500 \mathrm{mg}$ of soya lecithin were optimum characteristic than other formulations. They showed the average particles size $149 \pm 4.10 \mathrm{~nm}$ and PDI $250 \pm 2.04$. The zeta potential, \% EE and \% drug loading capacity was found to be respectively-29.7, 93.38 \pm 1.90 and $0.81 \pm 0.01$. The optimized batch of Paclitaxel SLNs exhibited spherical shape with smooth surface analyzed by Transmission Electron Microscopy. In vitro study showed sustained release profile and was found to follow Higuchi Kinetics Equation.

Conclusion: The SLNs of paclitaxel $\mathrm{m}$ et al. 1 the requirements of a colloidal drug delivery system. They had a particle size in nanosize; their size distribution was narrow and all the particles were in a spherical shape.

Keywords: Paclitaxel, Solid Lipid nanoparticles, Optimization, 32Full Factorial Design, Particle size, Transmission electron Microscopy

C 2019 The Authors. Published by Innovare Academic Sciences Pvt Ltd. This is an open access article under the CC BY license (http://creativecommons.org/licenses/by/4.0/) DOI: http://dx.doi.org/10.22159/ijap.2019v11i4.31134

\section{INTRODUCTION}

Paclitaxel is a diterpenoid pseudo alkaloid isolated from the Pacific Yew tree (Taxus brevifolia, Family Taxaceae) [1-5]. Paclitaxel has antineoplastic activity, being used against a wide spectrum of cancers. Paclitaxel is prescribed to treat most aggressive forms of ovarian, lung, head, neck, breast carcinoma and acute leukemia [6]. It is a taxane which interferes with microtubule depolymerization in tumor cells resulting in an arrest of the cell cycle in mitosis followed by induction of apoptosis [7]. However, the success of its clinical application is limited by its low therapeutic index and low water solubility due to its extreme hydrophobic character. The available marketed dosage forms of paclitaxel are Taxol $\AA^{\circledR}$ and Abraxane ${ }^{\circledR}$. Taxol $\AA$ is an intravenous infusion, which composed of 50:50 (v/v) cremophor EL (polyethoxylated castor oil) and dehydrated alcohol. Taxol shows serious side effects such as hypersensitivity, nephrotoxicity, neurotoxicity and cardiotoxicity attributable to paclitaxel or cremophor EL or both $[8,9]$. It is also cumbersome for the patients and limits the use of frequent dosing schedule for prolonged systemic exposure to the drug. To eliminate cremophor EL from paclitaxel formulation, many alternative cremophor EL free formulations have been investigated. Abraxane ${ }^{\circledR}$ is a cremophor EL free paclitaxel formulation and was registered with FDA in 2005. Its clinical profile was improved but generally, Abraxane ${ }^{\circledR}$ is not replacing Taxol@in cancer chemotherapy mostly due to its higher cost. Therefore to avoid the side effects and higher cost of the above formulations, there is a need to develop alternative nanocarriers system for paclitaxel [10].

Nanoparticles offer an alternative delivery system for cancer therapy that has the potential to control the release rate of drug, improve the drug pharmacokinetics and biodistribution, and reduce drug toxicity. Due to their small size, nanoparticles with entrapped drugs may penetrate tumors due to the discontinuous and leaky nature of the microvasculature of tumors $[11,12]$.

Lipid-based particulate delivery systems, like liposomes, micelles, nanocapsules, and solid lipid nanoparticles have been developed especially to solubilize poorly water-soluble and lipophillic drugs.
These lipid-based systems have the advantage that comprises bioderived and/or biocompatible lipids that often result in lower toxicity.

An experimental design is a statistical technique used to simultaneously analyze the influence of multiple factors on the properties of the system being studied. The purpose of experimental design is to plan and conduct experiments in order to extract the maximum amount of information from the collected data in the smallest number of experimental runs. Factorial design based on response surface method has been applied to design formulations $[13,14]$.

In the present study we investigated the possibility and potential of incorporating paclitaxel in SLNs made up of bio-acceptable and biodegradable lipid; stearic acid, using a microemulsion method and stabilized by a mixture of surfactants; soya lecitihin and poloxamer P-407 to produce stable and smaller sized nanoparticles with an aim to evaluate them as carrier for parenteral administration of paclitaxel. The effect of various concentrations of stearic acid and soya lecithin was analyzed for its influence on particle properties such as SLNs particle size, particle shape, zeta potential, entrapment efficiency, drug loading capacity and drug release profile.

\section{MATERIALS AND METHODS}

\section{Material}

Paclitaxel (Khandelwal Laboratories Pvt. Ltd. Thane), stearic acid, poloxamer P-407, soya lecithin, citric acid, potassium dihydrogen phosphate, disodium hydrogen phosphate, sodium chloride (Loba chemie Pvt. Ltd, Mumbai).

\section{Method}

In this study, A $3^{2}$ full factorial experimental design was used to optimize the formulation of nanoparticles. In the present investigation concentration of Lipid (X1) and concentration of Surfactant (X2) were selected as independent variables. The entrapment efficiency and particle size were selected as dependent variables. A statistical model incorporating interactive and polynomial terms was used to evaluate the response.

$$
\mathrm{Y}=\mathrm{b}_{0}+\mathrm{b}_{1} \mathrm{X}_{1}+\mathrm{B}_{2} \mathrm{X}_{2}+\mathrm{B}_{12} \mathrm{X}_{1} \mathrm{X}_{2}+\mathrm{B}_{11} \mathrm{X}_{12}+\mathrm{B}_{22} \mathrm{X}_{22}
$$


Table 1: Coding of actual values of variables

\begin{tabular}{|c|c|c|c|c|c|c|c|c|c|}
\hline Independent variable & P1 & P2 & P3 & P4 & P5 & P6 & P7 & P8 & P9 \\
\hline $\mathrm{X} 1$ & -1 & 0 & +1 & -1 & 0 & +1 & -1 & 0 & +1 \\
\hline $\mathrm{X} 2$ & -1 & -1 & -1 & 0 & 0 & 0 & +1 & +1 & +1 \\
\hline
\end{tabular}

X1: Lipid (stearic acid): 33.31 (1:20 milimoles), $66.62 \mathrm{mg}$ (1: 40 milimoles), 99.93 (1:60 milimoles), X2 Surfactant (soya Lecithin): 100 mg (1\%), 300 $\mathrm{mg}(3 \%), 500 \mathrm{mg}(5 \%)$

\section{Preparation of paclitaxel-loaded solid lipid nanoparticles}

The microemulsion of paclitaxel was prepared by melting stearic acid at $80^{\circ} \mathrm{C}$ to which paclitaxel was added and stirred for $5 \mathrm{~min}$. Soya lecithin was added to this mixture and stirred for $5 \mathrm{~min}$. Aqueous phase consisted of poloxamer P 407. Citric acid was added to maintain $\mathrm{pH} 5$ and heated at $80^{\circ} \mathrm{C}$. This mixture was then added to the melted lipid phase. This solution was kept on probe sonicator for a period of $10 \mathrm{~min}$ at 79 amplitude. Finally, o/w emulsion was formed. This microemulsion was carefully added dropwise into ice cold water present in the beaker with continuous stirring at $2000 \mathrm{rpm}$ on the magnetic stirrer. The SLN dispersion was stirred for $2 \mathrm{hr}$ after addition of microemulsion. This prepared microemulsion was further pass through HighPressure Homogenizer (HPH) keeping the pressure at 900 bars. The resultant SLN was characterized [15].

Table 2: Formulation of Paclitaxel SLNs

\begin{tabular}{|c|c|c|c|c|c|c|c|c|c|}
\hline \multirow[t]{2}{*}{ Ingredients (mg) } & \multicolumn{9}{|c|}{ Batches } \\
\hline & P1 & $\mathbf{P 2}$ & P3 & P4 & P5 & P6 & P7 & P8 & P9 \\
\hline Paclitaxel & $5 \mathrm{mg}$ & & & & & & & & \\
\hline Stearic Acid mg & 33.31 & 33.31 & 33.31 & 66.62 & 66.62 & 66.62 & 99.93 & 99.93 & 99.93 \\
\hline Poloxamer P $407 \mathrm{mg}$ & 28.59 & 28.59 & 28.59 & 57.18 & 57.18 & 57.18 & 85.77 & 85.77 & 85.77 \\
\hline Soya Lecithin mg & $\begin{array}{l}1 \% \\
(100)\end{array}$ & $\begin{array}{l}3 \% \\
(300)\end{array}$ & $\begin{array}{l}5 \% \\
(500)\end{array}$ & $\begin{array}{l}1 \% \\
(100)\end{array}$ & $\begin{array}{l}3 \% \\
(300)\end{array}$ & $\begin{array}{l}5 \% \\
(500)\end{array}$ & $\begin{array}{l}1 \% \\
(100)\end{array}$ & $\begin{array}{l}3 \% \\
(300)\end{array}$ & $\begin{array}{l}5 \% \\
(500)\end{array}$ \\
\hline
\end{tabular}

\section{Characterization of paclitaxel solid lipid nanoparticles \\ Particle size analysis}

The particle size and polydispersity index (PDI) of prepared solid lipid nanoparticles were determined using a Malvern Zetasizer ZS90 (Nano series Malvern Instrument). Each sample was diluted 10 times with distilled water to avoid multiscattering phenomena and is placed in disposable sizing cuvette and analyzed the particle size and PDI. The ploydispersibility index was studied to determine the narrowness of particle size distribution. The size analysis of sample consisted of 3 measurements and results were expressed as mean size \pm Std. Deviation [15].

\section{Zeta potential measurements}

Zeta potential is the potential difference between the dispersion medium and the stationary layer of fluid attached to the dispersed particle. Zeta potential is a key indicator of the stability of colloidal dispersions. The magnitude of the zeta potential indicates the degree of electrostatic repulsion between adjacent, similarly charged particle in the dispersion. The zeta potential of prepared Paclitaxel solid lipid nanoparticles was determined using a Malvern Zetasizer ZS90 (Nano series Malvern Instrument). The preconcentrated paclitaxel SLNs $1 \mathrm{ml}$ sample was diluted 10 times with distilled water in $10 \mathrm{ml}$ volumetric flask. From this solution, $1 \mathrm{ml}$ is placed in disposable zeta potential cuvette. The zeta limits ranged from- 200 to $+200 \mathrm{mV}$ [16].

\section{Determination of $\%$ entrapment efficiency}

Entrapment efficiency of Paclitaxel-loaded solid lipid nanoparticle was estimated by the centrifugation method. The cooling centrifuge was used. In this method prepared solid lipid nanoparticles were placed in centrifugation tube and centrifuged at $2000 \mathrm{rpm}$ for $20 \mathrm{~min}$ at $6-8^{\circ} \mathrm{C}$. The supernatant was withdrawn and pellets were washed with water. This was then kept in the cooling centrifuge at $15000 \mathrm{rpm}$ for $40 \mathrm{~min}$ at $6-8{ }^{\circ} \mathrm{C}$. The supernatant layer was removed and pellets were collected and diluted with methanol. By using UV spectrophotometer entrapped Paclitaxel was determined at $230 \mathrm{~nm}$. The samples from pellets were diluted before going for absorbance measurement. The amount of paclitaxel loaded in pellets gives the total amount of entrapped drug. The concentration of drug was calculated from the straight line equation obtained from the standard calibration curve of
Paclitaxel. Encapsulation efficiency is also expressed as a percent of drug trapped and was calculated using equation [17]

$\% E E=T$ otal amount Paclitaxel loaded in nanoparticles/total amount of drug used $X 100$

\section{\% Drug loading capacity}

Loading capacity (LC \%) can be calculated by the amount of total entrapped drug divided by the total nanoparticle weight. In drug delivery, yield, given as a percent, is a reflection of the amount of drug delivered per amount encapsulated. Loading capacity helps you to deal with nanoparticles after their separation from the medium and to know their drug content [18]. It is calculated by using the following equation;

$\% L C=[$ Entrapped Drug/Amount drug + Lipid + Excipient $] * 100$

\section{Shape and surface morphology}

Scanning Electron Microscopy (SEM) and Transmission Electron Microscopy (TEM) are very useful in determining the shape and morphology of lipid nanoparticles and allow determination of particle size and distribution. TEM determines the particle size with or without staining. SEM uses electrons transmitted from the specimen surface, while TEM uses electrons transmitted through the specimen. TEM allows visualization of SLNs after freeze fracturing and freeze substitution. Transmission Electron Microscopy was used to visualize the morphology of solid lipid nanoparticle. By using TEM, various characteristics like particle size, shape and internal structure of nanoparticulate carriers system can be efficiently evaluated.

\section{Procedure}

The surface morphology and shape of the optimized paclitaxel SLN were analyzed by TEM. The sample was diluted with double distilled water and then placed on copper grids coated with carbon film before negative staining with $2 \%(\mathrm{w} / \mathrm{v})$ sodium phosphotungstic acid solution for observation.

\section{In vitro drug release}

In vitro drug release was evaluated by using a dialysis bag diffusion technique. (Dialysis membrane with a molecular weight cut off $12,000)$. 


\section{The procedure of in vitro study}

The release of paclitaxel from the solid lipid nanoparticles was measured in triplicate in Phosphate Buffer Saline ( $\mathrm{pH}$ 7.4). In PBS pH $7.4,0.5 \%$ Tween was added. $2 \mathrm{mg}$ equivalent paclitaxel SLN was taken in a dialysis bag and both the ends of dialysis bag were closed and placed in a beaker containing $75 \mathrm{ml} \mathrm{PBS} \mathrm{pH} \mathrm{7.4.} \mathrm{This} \mathrm{dialysis} \mathrm{bag}$ was arranged in such a way that it just touches the surface of the buffer solution. The whole set up was placed on a magnetic stirrer which was rotated at 50 rpm. The temperature of buffer was maintained at $37 \pm 1{ }^{\circ} \mathrm{C} .2 \mathrm{ml}$ aliquots of release medium were withdrawn at a time interval $0.5,1,2,4,8,12,24$ and $48 \mathrm{~h}$. To maintain the sink condition, the same volume of dissolution media was added. The aliquots were centrifuged at 3000rpm for 5 to 10 min at room temperature. The supernatant was diluted with methanol and estimated by UV visible spectrophotometer at $230 \mathrm{~nm}$. The concentration of drug was calculated from the straight line equation obtained from standard curve of paclitaxel [19].

\section{RESULTS AND DISCUSSION}

Table 3: Effect of Stearic acid and soya lecithin on particle size, PDI, Zeta potential, \% EE and \%DLC

\begin{tabular}{lllll}
\hline Batch & Average particle size nm $^{*}$ & PDI $^{*}$ & Zeta potential (mV) & \% Entrapment efficiency* $^{*}$ \% Drug loading capacity $^{*}$ \\
\hline P1 & $152 \pm 1.1$ & $0.321 \pm 3.10$ & $-25.8 \pm 1.29$ & $75.29 \pm 3.20$ \\
P2 & $154 \pm 2.1$ & $0.415 \pm 1.35$ & $-27.7 \pm 2.87$ & $80.39 \pm 2.66$ \\
P3 & $149 \pm 4.1$ & $0.250 \pm 2.04$ & $-29.7 \pm 0.77$ & $93.38 \pm 1.90$ \\
P4 & $156 \pm 4.0$ & $0.333 \pm 1.50$ & $-24.3 \pm 1.12$ & $79.88 \pm 2.61$ \\
P5 & $159 \pm 4.9$ & $0.425 \pm 2.10$ & $-23.8 \pm 1.65$ & $84.17 \pm 2.36$ \\
P6 & $166 \pm 1.5$ & $0.355 \pm 3.20$ & $-22.6 \pm 1.10$ & $94.23 \pm 2.76$ \\
P7 & $259 \pm 4.5$ & $0.321 \pm 2.75$ & $-21.7 \pm 3.5$ & $80.79 \pm 1.96$ \\
P8 & $262 \pm 2.8$ & $0.280 \pm 2.50$ & $-20.2 \pm 2.40$ & $84.65 \pm 2.25$ \\
P9 & $265 \pm 1.4$ & $0.259 \pm 1.25$ & $-18.7 \pm 2.51$ & $95.46 \pm 2.33$ \\
\hline
\end{tabular}

All the values are expressed as mean $\pm \mathrm{SD}, \mathrm{n}=3$

The particle size is the most important property for in vivo integrity and biological fate of nanoparticles. The average particle size of paclitaxel SLNs were found in between $149 \pm 4.1 \mathrm{~nm}$ and $265.0 \pm 1.40 \mathrm{~nm}$. Fig. 1 shown the graph of particle size of Batch P3 which was best of an average value. The polydispersity indices of all batches reveal that all the batches have uniform particle size distribution. Batch P3 shows that Average particle size of $149 \pm 4.1$ and PDI of $0.250 \pm 2.04$.

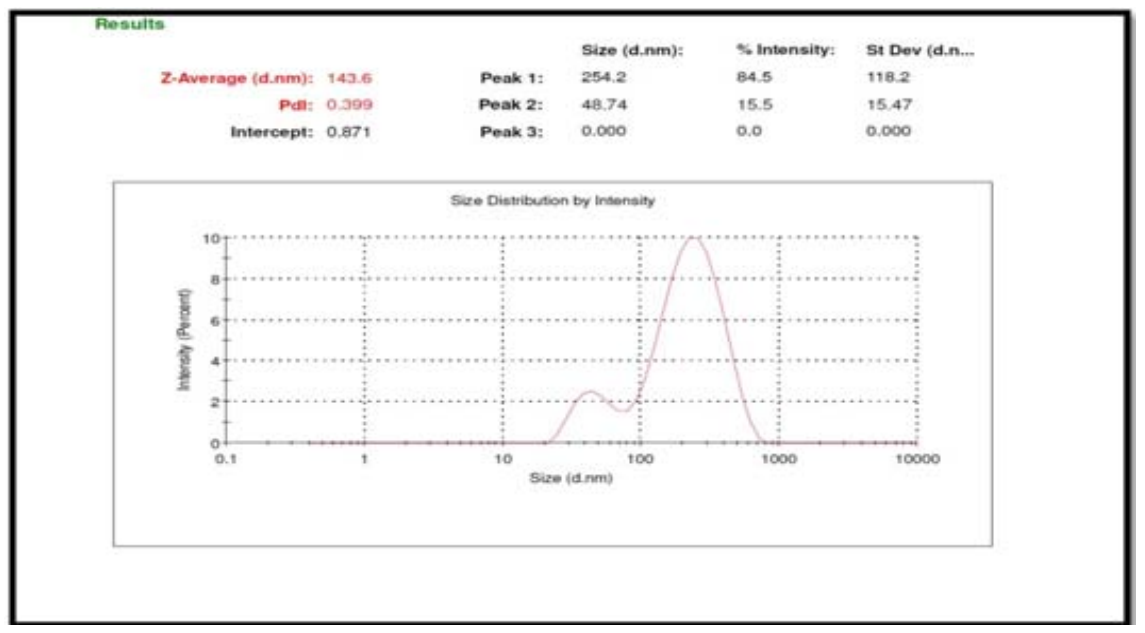

Fig. 1: Particle size distribution of batch P3

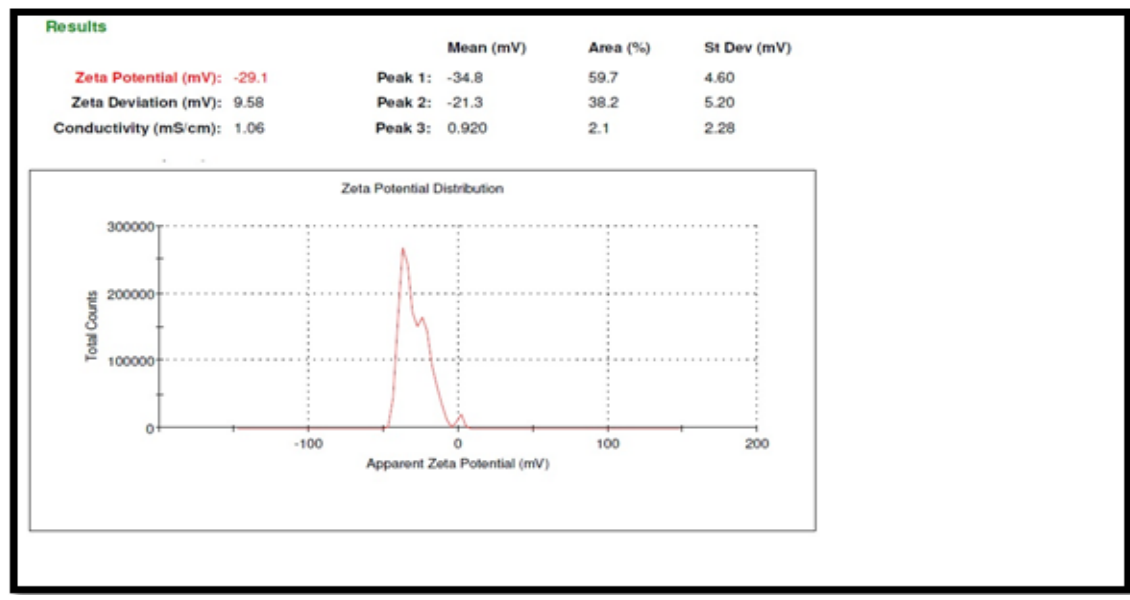

Fig. 2: Zeta potential of batch P3 
The stability study of prepared solid lipid nanoparticles was evaluated by measuring the zeta potential of nanoparticles using zeta meter. Zeta potential of P3 batch was-29.7 which indicates no agglomeration of the particles hence moderate stability. The increased concentrations of soya lecithin have not affected the zeta potential as revealed by the zeta potentials of the batches P1, P2 and P3. As the concentration of stearic acid is increased from batch P4 to P9, the zeta potential went on decreasing. Fig. 2 shown the zeta potential graph of batch P3 which was best of an average value.

Drug entrapment efficiency is an important property in drug-loaded nanocarriers and directly affects the therapeutic effect of system.
The entrapment efficiencies of all the batches have been shown in table 3 The results show that the amount of drug entrapped is increased with increase in the concentration of Stearic acid or soya lecithin. All the batches with highest concentration of Stearic acid (P3, P6 and P9) have shown highest entrapment efficiency. This may be attributed to the higher intactness of lipid. An increased concentration of soya lecithin increases entrapment efficiency by improving the film strength to increase the amount of drug in the nanoparticles. The highest entrapment was observed in batch P9 i.e. $95.46 \%$. The higher the encapsulation capacity of nanoparticles the larger the amount of drug released at the tumor site. The graphical representation was shown in fig. 3.

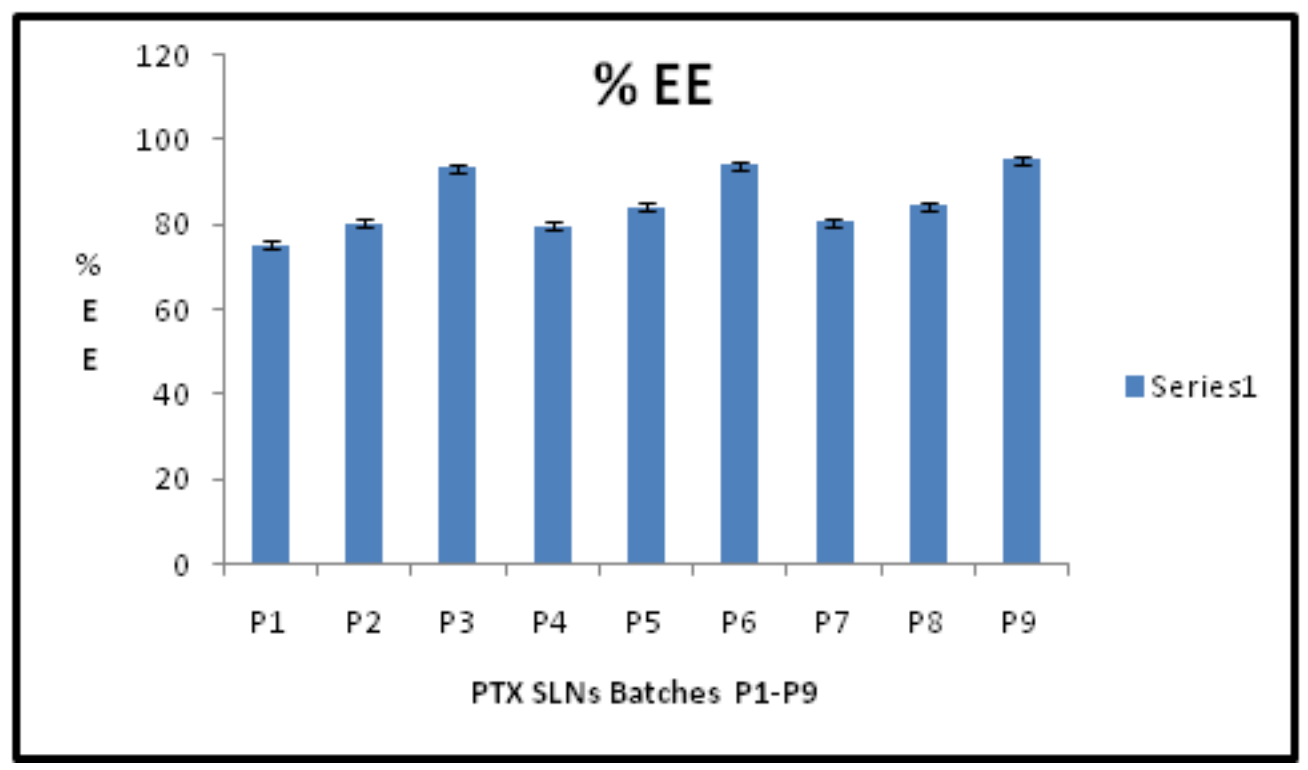

Fig. 3: Graphical representation of $\%$ entrapment efficiency (mean $\pm S D, n=3$ )

The batches P1, P2, P3, P4 and P7 have exhibited good drug loading capacities, their magnitude being above $0.8 \%$. Out of these batches P1, P2 and P3 batches produced the particles in the desired particle size range. However, the P1 and P2 batches produced nonhomogenous oily dispersions which led to their physical instability. Considering the parameters like particle size, drug loading capacity and physical stability of the formulation P3 batch has performed well in all aspects. Hence, it was treated to be optimized batch.

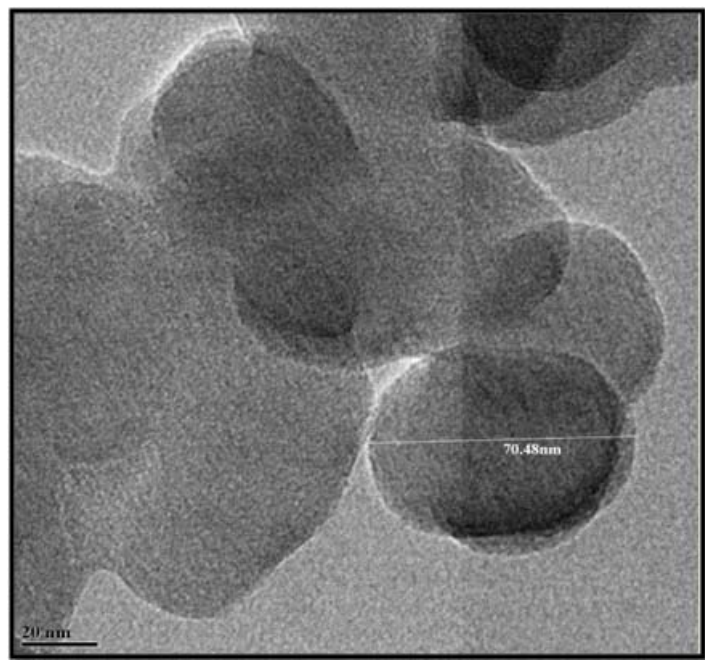

Fig. 4: TEM of Batch P3
The TEM study of optimized paclitaxel SLN illustrated the spherical shape of nanoparticles. The TEM image of the nanoparticles is depicted in fig. No. 4 It is evident from the image that the particles were spherical in shape with narrow size distribution and no aggregation. Particles of optimized paclitaxel SLN formulation were of nanosize. The surface of particles is smooth moreover their integrity was found to be intact.

\section{Optimization of paclitaxel SLNs}

\section{Data analysis of Y1 entrapment efficiency (\% EE)}

The \% EE of paclitaxel SLNs was determined by using ultracentrifugation method. The entrapment efficiency varied from $75.29 \pm 3.20 \%$ to $95.46 \pm 2.33 \%$. The results clearly indicated that Y1 is strongly affected by the amount of Lipid (stearic acid) and the amount of surfactant (soya lecithin) selected for the study. The response Y1 obtained at various levels of two independent variables was subjected to multiple regressions to give a quadratic polynomial equation.

The quadratic polynomial equation as follow:

$$
\mathrm{Y} 1=+84.06+1.97 \mathrm{~A}+7.85 \mathrm{~B}-0.8550 \mathrm{AB}-1.25 \mathrm{~A}^{2}+3.28 \mathrm{~B}^{2}
$$

\section{Contour plots and response surface analysis}

Two-dimensional contour plots and 3-D response surface plots for variables Y 1 (\% EE) are shown in fig. 6 and fig. 7 respectively. Fig. 6 and fig. 7 reveal an increase in entrapment efficiency (95.465) with an increase in the amount of lipid (stearic acid) and the amount of surfactant (soya lecithin) led to increases in \%entrapment efficiency. The highest entrapment efficiency was obtained with the highest amount of lipid. 


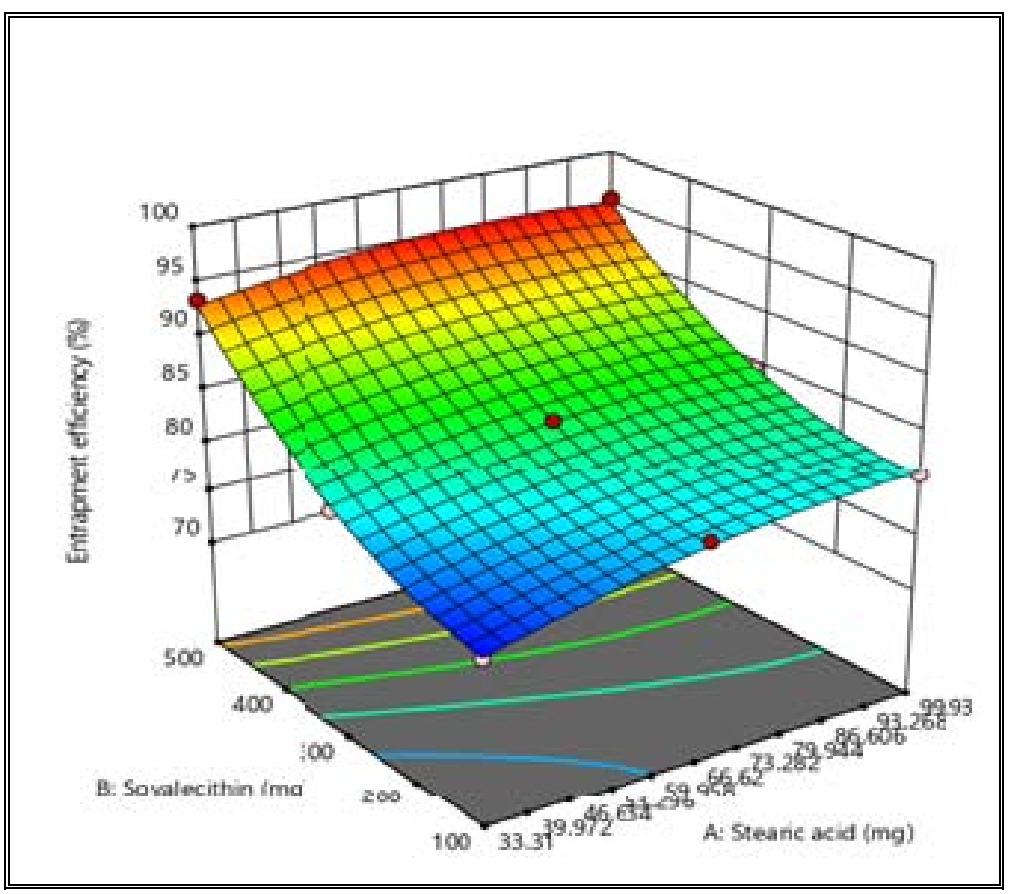

Fig. 5: Contour plot for Y1 (\% EE)

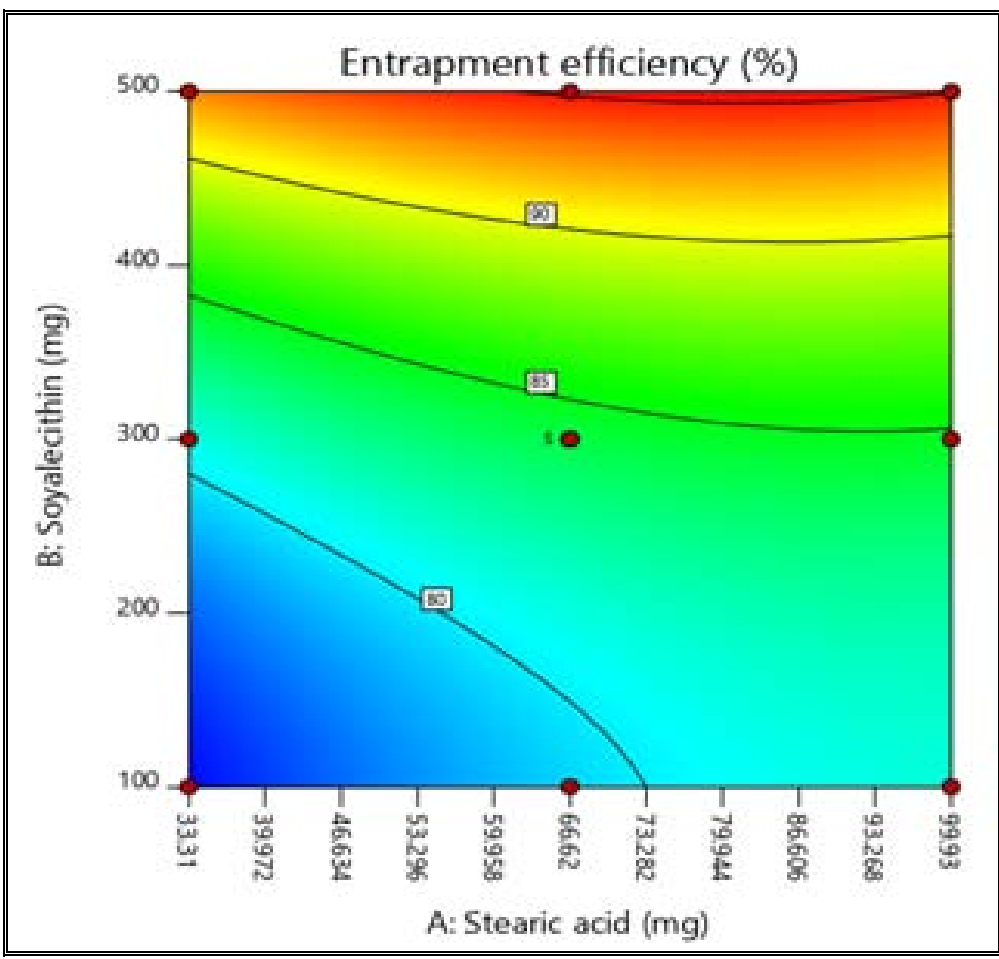

Fig. 6: Response surface plot for Y1 (\%EE)

\section{Data analysis of Y2 (Particle size)}

The particle size and PDI results of all the 9 batches of paclitaxel SLNs are tabulated in table 5. 22. The particle size of P1-P9 Batches was found in the range of $149.53 \pm 4.14$ to $265.06 \pm 1.40$ and the PDIs were in the range of $0.250 \pm 2.04$ to $0.425 \pm 2.10$. The results indicated an effect of the amount of stearic acid and soya lecithin on particle size. The response Y2 obtained to various levels of two independent variables was subjected in multiple regressions to give a quadratic polynomial equation.

$$
\mathrm{Y} 2=+159.95+55.07 \mathrm{~A}+2.13 \mathrm{~B}+2.24 \mathrm{AB}+47.27 \mathrm{~A}^{2}+0.2390 \mathrm{~B}^{2}
$$

\section{Contour plots and response surface analysis}

Two-dimensional contour plots and 3-D response surface plots for variables Y2 (Particle size) are shown in fig. 8and fig. 9 respectively. Fig. 8 and fig. 9 reveals that an increase in the amount of lipid led to an increase in particle size $(265.06 \pm 1.40 \mathrm{~nm})$. The lowest particle size was reported with the highest amount of surfactant and the lowest amount of lipid reveal a decline in particle size $(149.53 \pm 4.14$ $\mathrm{nm}$ ) with an increase in the amount of surfactant. But the effect of surfactant (soya leithin) was less on particle size Y2 than the effect of Lipid (stearic acid). 


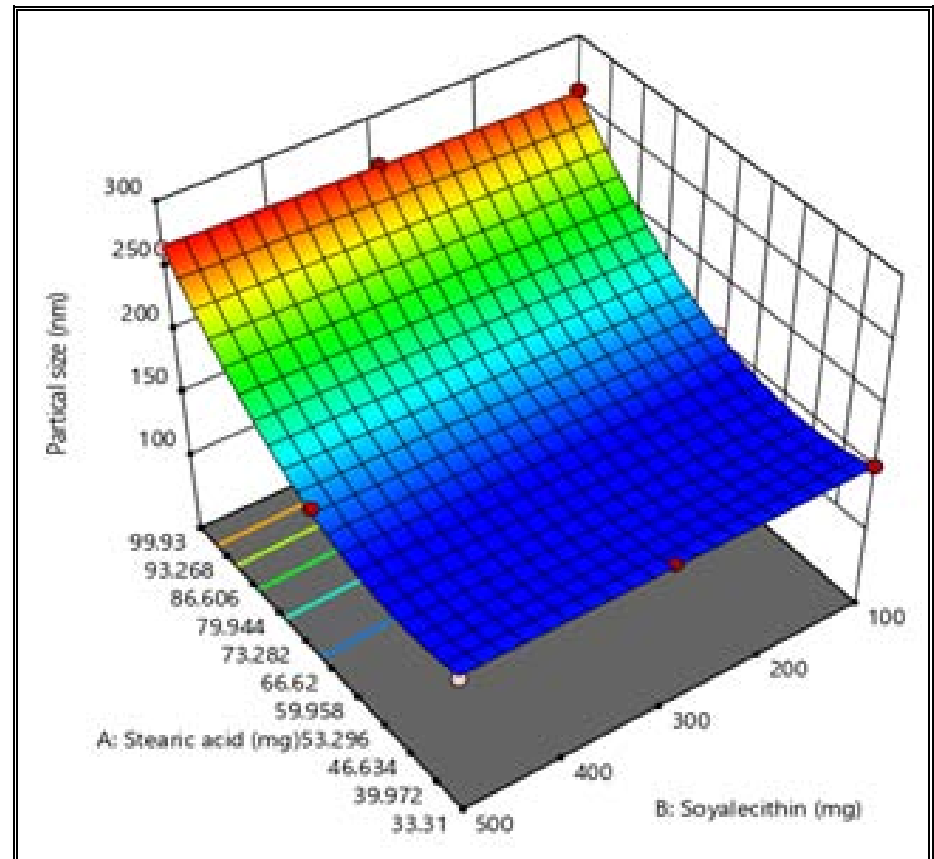

Fig. 7: Response surface plot for Y2 (Particle size)

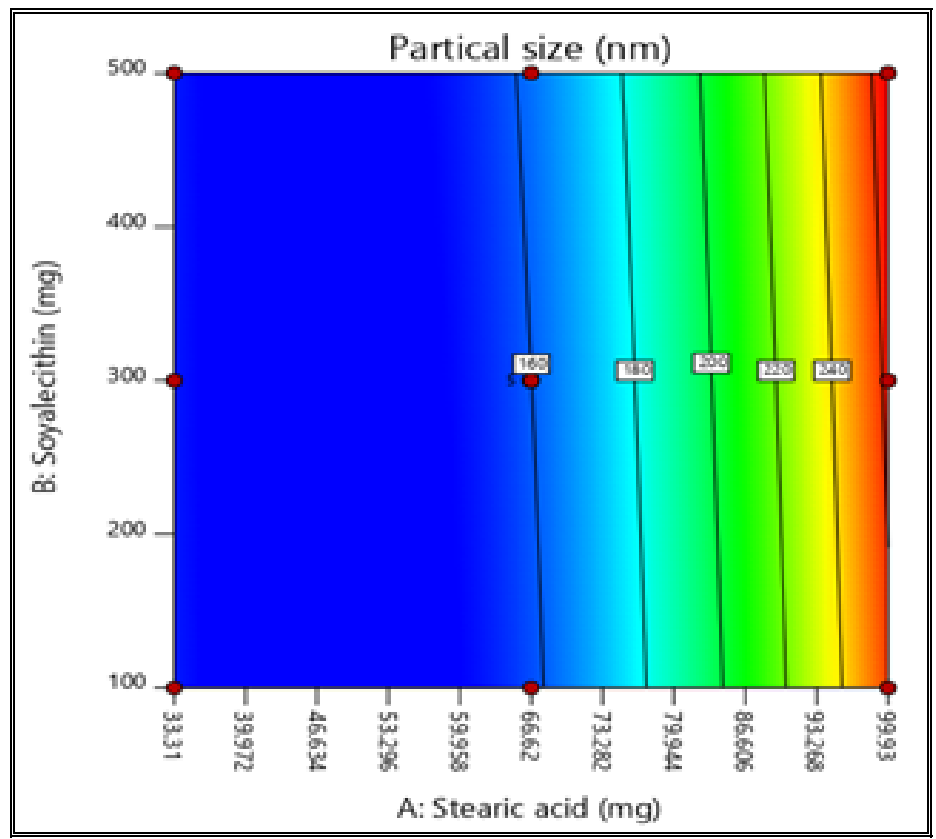

Fig. 8: Contour plot for Y2 (Particle size)

\section{Optimization}

The final optimal experimental parameter was calculated using optimization technique in the design expert softwares. This is used after generating the model polynomial equation which is to relate the dependent and independent variables. The design expert software was used which allows to compromise among the various responses and combination of factor level that jointly optimize the set of response by satisfying the requirement of each response in the set.

Table 4: Result of optimized batch P3

\begin{tabular}{llll}
\hline S. No. & Response & Experimental value & Predicted value \\
\hline 1 & \% Entrapment Efficiency & 93.38 & 94.17 \\
2 & Particle Size & 149.53 & 145.06 \\
\hline
\end{tabular}


Table 5: Formula of optimized batch P3

\begin{tabular}{lll}
\hline S. No. & Ingredients & Quantity \\
\hline 1. & Paclitaxel & $5 \mathrm{mg}$ \\
2. & Stearic acid & $33.31 \mathrm{mg}$ \\
3. & Soya lecithin & $500 \mathrm{mg}$ \\
4. & Poloxamer (407) & $28.50 \mathrm{mg}$ \\
5. & Distilled Water & $20 \mathrm{ml}$ \\
\hline
\end{tabular}

Table 6: Evaluation parameter of optimized batch P3 of PTX SLN

\begin{tabular}{lll}
\hline S. No. & Ingredients & Quantity \\
\hline 1. & \% Entrapment Efficiency & 93.38 \\
2. & Particle Size & $149.53 \pm 4.14$ \\
3. & PDI & $0.250 \pm 2.04$ \\
4. & Zeta potential & $-29.7 \pm 0.77$ \\
5. & \% DLC & $0.81 \pm 0.01$ \\
6. & In vitro drug release & $78.68 \pm 1.87$ \\
\hline
\end{tabular}

*The value are expressed as mean $\pm \mathrm{SD}, \mathrm{n}=3$

\section{In vitro drug release of optimized paclitaxel solid lipid nanoparticles}

In vitro drug release study of paclitaxel nanoparticles of optimized batch, P3 was studied by dialysis bag approach in PBS pH7.4 with tween 80 . The weight of prepared Paclitaxel SLN equivalent to $2 \mathrm{mg}$ drug was placed in the activated dialysis bag which is kept in phosphate buffer saline $\mathrm{pH} 7.4$ and performed in vitro drug release study. UV method was used for analyzing the data instead of HPLC which was used already. (15) The sustained release of paclitaxel with an initial burst release effect from lipid delivery system was observed. The sustained release of paclitaxel from nanoparticles may be the effect of localization of drug within the lipid matrix. Stearic acid being more lipophilic had sustained the release. The poloxamer, used as a surfactant, coated the nanoparticles. This coat prevented the degradation of nanoparticles in an acidic environment and the structural integrity was maintained. The \% cumulative drug release observations are tabulated in table 7.

Treatment of in vitro drug release data with different kinetic equations

The in vitro release study of optimized paclitaxel SLN was performed using a dialysis bag approach in PBS pH 7.4. The data of the in vitro drug release was used in different kinetic release pattern and the in vitro kinetic profile is tabulated in table 5 .

Table 7: In vitro drug release profile of optimized paclitaxel SLN

\begin{tabular}{lll}
\hline S. No. & Time (h) & Average percent cum \\
\hline 1. & 0 & 0 \\
2. & 0.5 & $6.24 \pm 1.89$ \\
3. & 1 & $11.96 \pm 2.31$ \\
4. & 2 & $17.31 \pm 3.43$ \\
5. & 4 & $24.86 \pm 2.95$ \\
6. & 8 & $40.83 \pm 2.68$ \\
7. & 12 & $51.97 \pm 2.74$ \\
8. & 24 & $64.22 \pm 2.80$ \\
9. & 48 & $78.68 \pm 1.87$ \\
\hline
\end{tabular}

${ }^{*}$ The value are expressed as mean $\pm \mathrm{SD}, \mathrm{n}=3$

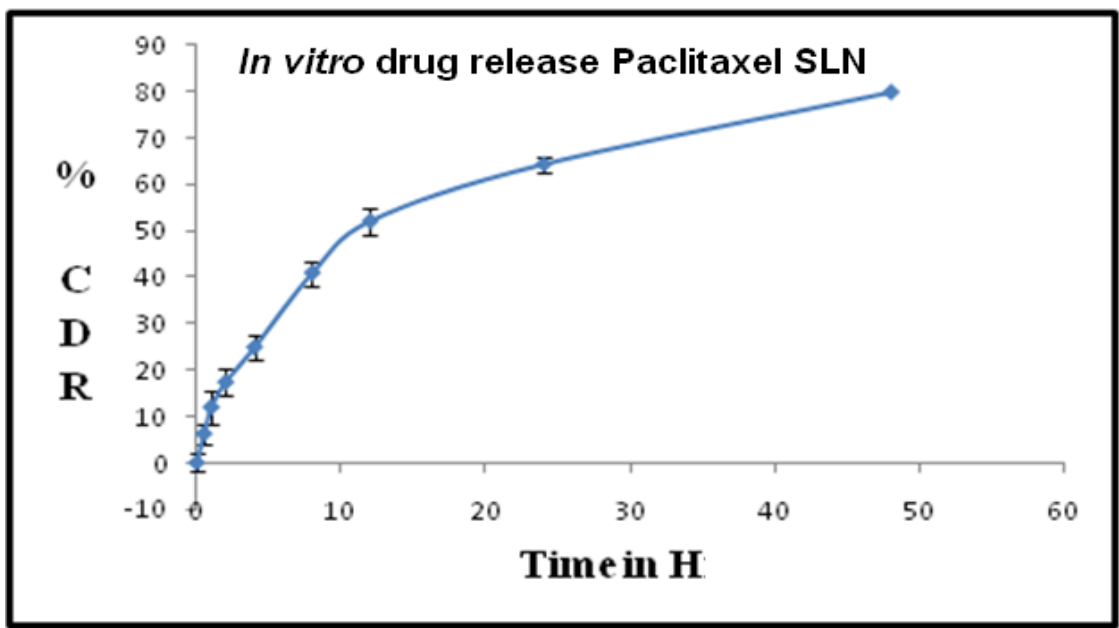

Fig. 9: In vitro drug release profile of batch $\mathrm{P} 3$ mean $\pm S D, n=3$ 
Table 5: In vitro drug release profile treated with kinetic equations

\begin{tabular}{llllll}
\hline S. No. & Time & Cumulative \% drug release & Log \% amt release & \% drug remaining & Log \% drug remaining \\
\hline 1. & 0 & 0 & 0 & 0.795 & 100 \\
2. & 6.24 & 1.077 & 93.76 & 1.972 \\
3. & 0.5 & 11.96 & 1.238 & 82.69 & 1.945 \\
4. & 1 & 17.31 & 1.395 & 75.14 & 1.917 \\
5. & 4 & 24.86 & 1.611 & 59.17 & 1.876 \\
6. & 8 & 40.83 & 1.715 & 48.03 & 1.772 \\
7. & 12 & 51.97 & 1.807 & 35.78 & 1.682 \\
8. & 24 & 64.22 & 1.901 & 20.32 & 1.308 \\
9. & 48 & 78.68 & & & \\
\hline
\end{tabular}

Table 6: Model fitting

\begin{tabular}{lllll}
\hline $\mathbf{R}^{2}$ & Zero-order & Peppas & Higuchi & First order \\
\cline { 2 - 4 } & 0.8209 & 0.9772 & 0.9735 & 0.9511 \\
\hline
\end{tabular}

The formulation followed peppas plot equation. This indicates that the PTX nanoparticle followed more than one type of release phenomenon and it appeared to be by the non fickian diffusion control mechanism.

\section{CONCLUSION}

The SLNs of paclitaxel met al. 1 the requirements of a colloidal drug delivery system. They had a particle size in nanosize; their size distribution was narrow and all the particles were in a spherical shape. The percent entrapment efficiency and percent drug loading capacities were in the acceptable limits. Moreover, the in vitro release pattern revealed that they served the purpose of being a sustained drug delivery system.

\section{ACKNOWLEDGMENT}

Authors are grateful to Khandelwal Laboratories Pvt. Ltd. Thane for providing the gift sample of Paclitaxel. We are also thankful to the Management of Rajarambapu college of Pharmacy, Kasegaon for providing the necessary facilities to carry out this work.

\section{CONFLICT OF INTERESTS}

The authors declare no conflict of interest

\section{REFERENCES}

1. Wani M, Taylor $\mathrm{H}$, Wall $\mathrm{M}$, Coggon $\mathrm{P}, \mathrm{McPhail}$ A. Plant antitumor agents. VI. The isolation and structure of taxol, a novel antileukemic and antitumor agent from taxus brevifolia. J Am Chem Soc 1971;93:2325-7.

2. Hawkins MJ. New anticancer agents: taxol, camptothecin analogs, and anthrapyrazoles. Oncology 1992;6:17-23.

3. Holmes FA, Walters RS, Theriault RL, Forman AD, Newton LK, Raber MN, et al. Phase II trial of taxol, an active drug in the treatment of metastatic breast cancer. J Natl Cancer Inst 1991;83:1797-805.

4. Markman M. Taxol: an important new drug in the management of epithelial ovarian cancer. Yale J Biol Med 1991;64:583-90.

5. Sung FL, Poon TC, Hui EP, Ma BB, Liong E, To KF, et al. Antitumor effect and enhancement of cytotoxic drug activity by cetuximab in nasopharyngeal carcinoma cells. In Vivo 2005;19:237-45.

6. Spencer and Faulds, Paclitaxel: a review of its pharmacodynamic and pharmacokinetic properties and therapeutic potential in the treatment of cancer. Adis Int Limited Auckland New Zealand 1994;48:794-847.
7. Saville MW, Lietzau J, Pluda JM, Wilson WH, Humphrey RW, Feigel E, et al. Treatment of HIV associated kaposi's sarcoma with paclitaxel. Lancet 1995;346:26-8.

8. Trissel LA, Xu QA, Gilbert DL. Compatibility and stability of paclitaxel combined with doxorubicin hydrochloride in infusion solutions. Ann Pharmacother 1998;32:1013-6.

9. Zhang $Y, \mathrm{Xu}$ QA, Trissel LA, Gilbert DL, Martinez JF. Compatibility and stability of paclitaxel combined with cisplatin and with carboplatin in infusion solutions. Ann Pharmacother 1997;31:1465-70.

10. Xiaowei Dong A, Cynthia A Mattingly a, Michael Tseng b, Moo Cho c, Val R Adams a, Russell J. Mumper, Development of new lipid-based paclitaxel nanoparticles using sequential simplex optimization. Eur J Pharm Biopharm 2009;72:9-17.

11. R Pasqualini, W Arap, DM McDonald. Probing the structural and molecular diversity of tumor vasculature. Trends Mol Med 2002;8:563-71.

12. SK Hobbs, WL Monsky, F Yuan, WG Roberts, L Griffith, VP Torchilin, et al. Regulation of transport pathways in tumor vessels: role of tumor type and microenvironment. Proc Natl Acad Sci USA 1998;95:4607-12.

13. MC Gohel, AF Amin. Formulation optimization of controlled release diclofenac sodium microspheres using factorial design. J Controlled Release 1998;51:115-22.

14. MD Bhavsar, SB Tiwari, MM Amiji. Formulation optimization for the nanoparticles-in-microsphere hybrid oral delivery system using factorial design. J Controlled Release 2006;110:422-30.

15. A Dinda, I Biswal, P Chowdhury, R Mohapatra. Formulation development and evaluation of paclitaxel-loaded solid lipid nanoparticles using glyceryl monostearate. J Appl Pharm Sci 2013;3:133-8.

16. D Pandita, A Ahuja, T Velpandian, V Lather, T Dutta, RK Khar. Characterization and in vitro assessment of paclitaxel-loaded lipid nanoparticles formulated using modified solvent injection technique. Pharmazie 2009;64:5.

17. Sidharth M Patil, Hemant $P$ Joshi, Research Journal of Pharmaceutical. Optimizing the concentration of ampiphiles in the development of colloidal drug carriers for paclitaxel. Biol Chem Sci 2013;4:1066-80.

18. Hoo Kyun Choia b, Robhash Kusam Subedi a, Keon Wook Kanga. Preparation and characterization of solid lipid nanoparticles loaded with doxorubicin. Eur J Pharm Sci 2009;37:508-13.

19. Rita R Lala, Amol S Shinde, Nikita Y. Nandvikar solid lipid nanoparticles: a promising approach for combinational drug therapy in cancer. Int J Appl Pharm 2018;10:17-22. 